[5] de Vlam K, Mielants H, Cuvelier C, de Keyser F, Veys EM, de Vos M. Spondyloarthropathy is underestimated in inflammatory bowel disease: prevalence and HLA association. J Rheumatol Dec 2000;27(12):2860-2865.

Disclosure of Interest: None declared

DOI: 10.1136/annrheumdis-2017-eular.2228

\section{AB1020 MULTIFREQUENCY BIOIMPEDANCE COMBINED WITH ACOUSTIC MYOGRAPHY - A NON-INVASIVE PAIN-FREE ASSESSMENT OF MUSCLE USE}

E.M. Bartels, J. Kvistgaard Olsen, H. Bliddal, L.E. Kristensen,

B. Danneskiold-Samsøe. The Parker Institute, Department of Rheumatology, Copenhagen University Hospital, Bispebjerg and Frederiksberg, Frederiksberg, Denmark

Background: Musculoskeletal diseases may involve muscle function, which often deteriorates due to a combination of pain and lack of exercise. Possible correction of this by training, or at least achievement of optimal efficiency of involved muscles with therapy, taking age and affecting disease into account, is an area which needs easily applicable and non-invasive pain-free assessment methods capable of monitoring daily living tasks outside a strict laboratory setting. Multi-frequency bioimpedance ( $\mathrm{mfBIA}$ ), assessing muscle health prior to exercise, in combination with Acoustic Myography (AMG), which allows real-time tests while e.g. walking or bicycling, may be such a method.

Objectives: To validate AMG combined with mfBIA for muscle-use assessment during a series of daily activity movements and exercise with the aim of introducing the method in the clinic.

Methods: 10 healthy subjects aged 25-68 years were assessed with mfBIA (Impedimed, Brisbane, Australia) prior to and following exercise of $\mathrm{m}$. gastrocnemius during walking, stair climbing and descending, and cycling with increasing load. AMG was recorded with a CURO unit (MyoDynamik ApS, Frederiksberg, Denmark), and data handling was carried out with the software belonging to the devices. The mfBIA parameters considered were resistance $(R)$, internal $(R i)$ and external $(\mathrm{Re})$ resistance and centre frequency (fc), and the AMG parameters were Efficiency $(E)$ - synchronization of motoric units, Temporal summation $(T)$ - how frequently do you use a particular muscle fibre, and Spatial summation (S) - how many fibres in use at a given time, all given as Median; Min,Max.

Results: The mfBIA data, showing the health state of the muscle, showed changes in $\mathrm{R}, \mathrm{Ri}, \mathrm{Re}$ and fc as expected for a healthy muscle as an effect of exercise. The AMG data showed good reproducibility with repeated measurements. Walking on flat ground was less synchronized $(E 2 ; 1,4)$ as were walking up $(E 4 ; 1,6)$ and down stairs $(E 3 ; 1,6)$, than cycling $(E 8.5 ; 5,9)$. With increasing load during cycling, $E$ decreased with the higher demand to $E 5.5$ range 1-7. The T-score was similar around 7 for all types of walking, while overall decreasing with increasing load during cycling from 6.5 to 5.5. The S-score was low during the three types of walk, indicating use of many fibres ( $S$ around 3 ), which is in line with the low E-score. For cycling the S-score decreased slightly with increasing load, from 8 to 7.5 . Conclusions: The combined method of mf-BIA and AMG shows good reproducibility. This method has the potential to assess training possibilities in patients with musculoskeletal diseases by testing directly on muscles during the movements of daily function. The method is applicable in real life settings outside the laboratory.

Acknowledgements: The Parker Institute is supported by the OAK Foundation Disclosure of Interest: None declared

DOI: 10.1136/annrheumdis-2017-eular.3369

\section{AB1021 VIDEOCAPILLAROSCOPIC FINDINGS IN PATIENTS WITH SYSTEMIC LUPUS ERYTHEMATOSUS WITH OR WITHOUT JACCOUD'S ARTHROPATHY}

C. Lins ${ }^{1}$, E.P.D. Fonseca ${ }^{2}$, D.L.D.S. Ribeiro ${ }^{2}$, W.G.D. Santos ${ }^{2}$, G. Rosa ${ }^{2}$, V. Machicado ${ }^{2}$, A.L. Pedreira ${ }^{2}$, A.P.M.D. Souza ${ }^{2}$, C. Baleeiro ${ }^{2}$, L.G.D.S. Ferreira ${ }^{2}$, I.S.D. Oliveira ${ }^{3}$, J.P.C.G.D. Silva ${ }^{3}$, A.M. Atta $^{3}$, M.B. Santiago ${ }^{2} .{ }^{1} E B M S P$ - Escola Bahiana de Medicina e Saude Publica ${ }^{2}$ EBMSP - Escola Bahiana de Medicina e Saude Publica; ${ }^{3}$ HUPES - Hospital Universitario Edgard Santos, Salvador, Bahia, Brazil

Background: Systemic lupus erythematosus (SLE) is an autoimmune disease that can present, as other collagen vascular disorders, changes in blood vessels. It can be evaluated by a non-invasive technique called periungual nailfold videocapillaroscopy (VCP). This technique is helpful in the diagnosis of systemic sclerosis (SSc), being part of the new classification criteria, and identifies individuals with Raynaud's phenomenon who are at a higher risk for developing SSc.

Objectives: This study aims to describe the videocapillaroscopic profile of a series of SLE patients and investigate if the VCP pattern is different among those with Jaccoud's arthropathy (JA) compared to the patients without this complication

Methods: The patients were submitted to VCP, clinical evaluation, and laboratory tests. The capillaroscopic patterns were defined as minor, major and scleroderma (SD). The presence of capillaroscopic findings such as elongated capillaries, increased tortuosity, ectasia, prominent venous plexus, neoangiogenesis, hemorrhage and megacapillaries was also observed.

Results: In a population of 113 female patients with SLE (67 without JA and 46 with JA), at least one alteration was observed in VCP in $89.40 \%$ of patients, and the "nonspecific changes" were the most prevalent. Minor changes were seen in $39(58.2 \%)$ and $26(56.5 \%)$ patients, and major changes were seen in $21(31.3 \%)$ and $11(23.9 \%)$ patients without and with JA, respectively. The SD patterns were observed in $02(3.0 \%)$ and $03(6.5 \%)$ patients without and with JA, respectively $(p>0.05)$.

Conclusions: The majority of patients of SLE present changes in the VCP exam, but such a tool does not allow distinguish those with or without JA.

References:

[1] Lambova SN, Muller-Ladner U. Capillaroscopic pattern in systemic lupus erythematosus and undifferentiated connective tissue disease: what we still have to learn? Rheumatology international. 2013;33(3):689-95.

[2] Ingegnoli F. Capillaroscopy abnormalities in relation to disease activity in juvenile systemic lupus erythematosus. Microvascular research. 2013;87:92-4. Santiago MB, Galvao V. Jaccoud arthropathy in systemic lupus erythematosus: analysis of clinical characteristics and review of the literature. Medicine. 2008;87(1):37-44

[3] Ingegnoli F, Zeni S, Meani L, Soldi A, Lurati A, Fantini F. Evaluation of nailfold videocapillaroscopic abnormalities in patients with systemic lupus erythematosus. Journal of clinical rheumatology: practical reports on rheumatic \& musculoskeletal diseases. 2005;11(6):295-8.

[4] Ragab O, Ashmawy A, Abdo A, Mokbel A. Nailfold capilloroscopy in systemic lupus erythematosus. The Egyptian Rheumatologist. 2011;33:61-7.

Acknowledgements: M. B.S. has received a scholar ship from Conselho Nacional de Desenvolvimento Científico e Tecnológico (CNPq).

Disclosure of Interest: None declared

DOI: 10.1136/annrheumdis-2017-eular.6627

\section{AB1022 THE RECALL SURVEY: THE RELATIONSHIP BETWEEN ULTRASOUND SYNOVITIS AND BONE EROSION IN PATIENTS} WITH RHEUMATOID ARTHRITIS

E. Filippucci ${ }^{1}$, C. Bonali ${ }^{2}$, P. Macchioni ${ }^{3}$, A. Iagnocco ${ }^{4}$, A. Delle Sedie ${ }^{5}$ S. Tropea ${ }^{6}$, O. Epis ${ }^{7}$, M. Canzoni ${ }^{8} .{ }^{1}$ Clinica Reumatologica, Università Politecnica delle Marche, Ancona; ${ }^{2}$ Rheumatology Unit, Ospedale San Paolo, Bari; ${ }^{3}$ Rheumatology Unit, Reggio Emilia Hospital, Reggio Emilia: ${ }^{4}$ Academic Rheumatology Unit, Università degli Studi di Torino, Torino; ${ }^{5}$ Rheumatology Unit, Università di Pisa, Pisa; ${ }^{6}$ ASP7, Busacca Hospital, Ragusa; ${ }^{7}$ Rheumatology Unit, Ospedale Niguarda Milano, Milano; ${ }^{8}$ Local Health Unit (ASL) Rome-1, Rome, Italy

Background: Ultrasound (US) has shown to be a sensitive imaging tool for the detection of subclinical signs of synovitis in patients (pts) with rheumatoid arthritis (RA); further studies are still required to delineate the impact of US findings in the management of RA pts in daily clinical practice.

Objectives: To investigate the relationship between US findings indicative of joint inflammation and US bone erosions at joint level in pts with RA.

Methods: In 2015 an educational event focused on the added value of US in RA pts was held in 22 rheumatology centers in Italy. In every center, the local rheumatologists provided RA pts to be examined by US. Pts signed an informed consent and a brief history of them was collected by the local rheumatologists (previous and current therapy, DAS28, HAQ score). Bilateral US examinations of wrists, metacarpophalangeal (MCP) and metatarsophalangeal (MTP) joints were performed by rheumatologists expert in US, to assess synovitis (joint effusion, synovial proliferation, and power Doppler (PD) signal), and bone erosions, using a Logiq E R7, General Electrics, with a 4.2-13 MHz linear probe. All US findings were scored using a 4 degree semiquantitative scoring system.

Results: In 465 RA pts, a total of 10.230 joints were scanned. Of these joints, 3.969 (39\%) showed joint effusion and/or synovial proliferation and $1.784(17 \%)$ were found positive for PD signal. The most frequently involved joints were the wrists followed by the second MCP joints and first MTP joints. In 749 joints US detected at least one bone erosion. The most frequently eroded joints were the wrists, the second and fifth MCP joints and the first and fifth MTP joints. A total of 226 RA pts showed at least one bone erosions and in $181(80 \%)$ of these pts the eroded joints were found positive for PD signal.

Conclusions: A high prevalence of PD signal was found in the joints found eroded by US. This is the first study providing such an evidence using a portable US equipment.

Disclosure of Interest: None declared

DOI: 10.1136/annrheumdis-2017-eular.4286

\section{AB1023 QUANTIFERON ${ }^{\circledR}$-TB GOLD IN-TUBE ASSAY CAN BE USED FOR LATENT TUBERCULOSIS SCREENING BEFORE BIOLOGICAL DRUG TREATMENT IN A BCG VACCINATED COUNTRY: HUR-BIO SINGLE CENTER REAL LIFE RESULTS}

E. Seyhoğlu ${ }^{1}$, O.A. Uyaroğlu ${ }^{1}$, A. Erden ${ }^{2}$, L. Kılıç ${ }^{2}$, B. Armağan ${ }^{2}$, A. Sarı ${ }^{2}$, M. Baykal ${ }^{2}$, S. Ak ${ }^{2}$, Ö. Karadağ ${ }^{2}$, A. Akdoğan ${ }^{2}$, S. Apraş Bilgen ${ }^{2}$, S. Kiraz ${ }^{2}$, I. Ertenli ${ }^{2}$, U. Kalyoncu ${ }^{2} .{ }^{1}$ Department of Internal Medicine; ${ }^{2}$ Division of Rheumatology, Department of Internal Medicine, Hacettepe University School of Medicine, Ankara, Turkey

Background: Patients treated with biologic agents have increased risk of 
developing active tuberculosis (TB). It is essential to screen patients for latent TB before starting a biologic drug. Population is vaccinated with BCG in Turkey and $B C G$ vaccination decreases the specifity of tuberculin skin test. QuantiFERON-TB Gold In-Tube Assay (QFT) is an another good option to screen latent TB.

Objectives: The objective of this study was to assess reliability of QFT test for latent TB before biological treatment.

Methods: Hacettepe University Rheumatology Biologic Registry (HUR-BIO) is a single center biological registry since 2005. Between Nov 2011 and July 2015, 1347 patients were assessed by QFT for latent TB. All consecutive patients were evaluated by a standard questionnaire between July 2015 and October 2016. This questionnaire included demographic characteristics, medical and treatment history, symptoms of active TB. 671 patients were assessed by the physicians. TB status of other 676 patients were checked from Turkish national tuberculosis registery records. It's an obligatory disposition for physicians to inform health ministery about TB cases and all TB patients must be recorded in those regitry. The mean TB incidance per year was calculated for every anti-TNF agents and non-TNF biological agents.

Results: Total 1347 (58.1\% female) patients were recruited to study. Mean age was $42 \pm 12$ years. Diagnosis were followed; RA 436 (32.4\%), SpA 844 $(62.5 \%)$, others $67(5.1 \%)$. Total biological drug exposure was 2329 patientyears; adalimumab (660 years), etanercept (630 years), infliximab (426 years), golimumab (283 years), certolizumab (78 years), and total anti-TNF duration (2071 years). Non-TNFi exposure was 258 patient-years. Positive and indeterminate QFT results were found in $267(19.8 \%)$ and $20(1.5 \%)$ patients, respectively and those patients were prescribed INH prophylaxis. In addition, INH was prescribed to $37(2.7 \%)$ patients according to chest X-Ray and physician decision. Pulmonary TB found in 3 of $1347(0.22 \%)$ patients. TB was developed 38,28 and 21 months after TNFi. The mean TB incidance per year was $128.8 / 100.000$ for all biological drugs. The mean TB incidance per year according to QFT positive and negative patients were $181.8 / 100.000$ vs $112.4 / 100.000$.

Conclusions: According to QFT screening for latent TB, INH was started almost $20 \%$ of patients. However, if we used TST for latent TB test in BCG vaccinated countries, INH would started almost $70-80 \%$ of patients. Therefore QFT was a good tool for latent TB screening in BCG vaccinated countries. Consequently, QFT test seems acceptable to determinate latent TB during biological drug usage. In addition, TB incidance has increased almost 7 times of our national TB incidance. Disclosure of Interest: None declared

DOI: 10.1136/annrheumdis-2017-eular.4080

\section{AB1024 ULTRASOUND IN GIANT CELL ARTERITIS: CUT-OFF AND PITFALLS IN THE HALO SIGN}

E. De Miguel ${ }^{1}$, L.M. Beltran ${ }^{2}$, I. Monjo ${ }^{1}$, F. Deodati ${ }^{2}$, W.A. Schmidt ${ }^{3}$,

J. García-Puig ${ }^{2} .{ }^{1}$ Rheumatology; ${ }^{2}$ Internal Medicine, Hospital Universitario la Paz, Madrid, Spain; ${ }^{3}$ Rheumatology, Immanuel Krankenhaus, Berlin, Germany

Background: At the age of presentation of Giant Cell Arteritis (GCA) atherosclerosis is common. The ultrasonographic (US) appearance of athermanous plaque is usually easily differentiated from the hypoechoic halo of GCA. However, the US appearance of the increased of the intima-media-thickness (IMT) in an atherosclerotic carotid artery may have similar image appearance as the halo sign. We hypothesize that atherosclerosis could produce an increase of temporal artery (TA) IMT and cause false-positives halo sign.

Objectives: The aim of this study was to explore the better cut-off in the IMT of TA to minimise the number of false-positive GCA diagnosis caused by atherosclerosis.

Methods: Consecutive non selected patients, $\geq 50$ years-old with high vascular risk according to European Guidelines on cardiovascular disease prevention, and without signs or symptoms of GCA, were included.

Ultrasonography of carotid artery: Carotid US examinations were performed on a Mylab Seven (Esaote Medical Systems, Italy) with a 4-13 MHz linear-array. The system employed dedicated software radiofrequency-tracking technology to obtain IMT $\left(\right.$ QIMT $\left.^{\circledR}\right)$.

Ultrasonography of temporal superficial artery: A color Doppler ultrasound (CDU) and grey scale measure of the IMT/halo sign in both TA and its branches was performed by a second experienced sonographer. A Mylab Twice equipment (Esaote, Geneve, Italy) was used, with a $22 \mathrm{MHz}$ frequency for grey scale and a $12.5 \mathrm{MHz}$ for CDU (color gain of 51, PRF of $2 \mathrm{kHz}$ ). The sonographer was blind to the clinical and carotid ultrasound IMT data.

Results: Forty patients were studied, 28 men $(70 \%)$, with a mean age of $70,6 \pm 6,9$ years. Three patients were active smokers and 27 ex-smokers. Arterial hypertension was present in $39(97.5 \%)$, dyslipidaemia in $34(85 \%)$ and diabetes in $19(47.5 \%)$. The mean erythrocyte sedimentation rate was $13.6 \pm 11.0$. The table shows that an IMT $>0.30 \mathrm{~mm}$ (halo sign) was seen in at least 1 TA branch of 18 patients (45\%) with 33 TA branches affected $(20.6 \%)$. An IMT cut-off $>0.34$ $\mathrm{mm}$, was present in 4 patients $(10 \%)$. When at least two affected branches with this measure were required to make the US diagnosis (criteria recommended to improve specificity) only one patient $(2.5 \%)$ produced a false-positive halo sign.

Conclusions: To the best of our knowledge, this is the first communication indicating that atherosclerosis is a potential cause of false-positive halo sign. We propose a cut-off of AT IMT $>0.34 \mathrm{~mm}$ in at least two branches to minimise the number of false positives in GCA diagnosis.

Disclosure of Interest: None declared

\begin{tabular}{|c|c|c|c|c|c|c|c|}
\hline & \multirow{2}{*}{$\begin{array}{l}\text { Carotid } \\
\text { IMT mm }\end{array}$} & \multicolumn{2}{|c|}{ Right } & \multicolumn{2}{|c|}{ Left } & \multicolumn{2}{|c|}{ Number of branches with halo } \\
\hline & & $\begin{array}{l}\text { TA } \\
\text { frontal }\end{array}$ & $\begin{array}{c}\mathrm{TA} \\
\text { parietal }\end{array}$ & $\begin{array}{l}\text { TA } \\
\text { frontal }\end{array}$ & $\begin{array}{c}\text { TA } \\
\text { parietal }\end{array}$ & $\begin{array}{c}\text { Cut-off } \\
\text { IMT }>0.3 \mathrm{~mm}\end{array}$ & $\begin{array}{c}\text { Cut-off } \\
\text { IMT }>0.34 \mathrm{~mm}\end{array}$ \\
\hline Patient 4 & 1.185 & 0.37 & 0.31 & 0.31 & 0.31 & 4 & 1 \\
\hline Patient 5 & 0.948 & 0.26 & 0.31 & 0.31 & 0.27 & 2 & 0 \\
\hline Patient 6 & 1.135 & 0.18 & 0.31 & 0.24 & 0.25 & 1 & 0 \\
\hline Patient 7 & 1.164 & 0.31 & 0.28 & 0.28 & 0.28 & 1 & 0 \\
\hline Patient 9 & 1.243 & 0.37 & 0.4 & 0.45 & 0.35 & 4 & 4 \\
\hline Patient 10 & 1.196 & 0.28 & 0.28 & 0.34 & 0.28 & 1 & 0 \\
\hline Patient 11 & 1.21 & 0.31 & 0.31 & 0.25 & 0.28 & 2 & 0 \\
\hline Patient 12 & 1.165 & 0.29 & 0.28 & 0.28 & 0.31 & 1 & 0 \\
\hline Patient 14 & 1.37 & 0.29 & 0.22 & 0.31 & 0.31 & 2 & 0 \\
\hline Patient 16 & 1.33 & 0.25 & 0.28 & 0.23 & 0.34 & 1 & \\
\hline Patient 17 & 1.027 & 0.28 & 0.32 & 0.37 & 0.34 & 3 & 1 \\
\hline Patient 18 & 0.989 & 0.25 & 0.31 & 0.28 & 0.28 & 1 & 0 \\
\hline Patient 19 & 1.017 & 0.19 & 0.28 & 0.25 & 0.31 & 1 & 0 \\
\hline Patient 25 & 0.802 & 0.29 & 0.27 & 0.31 & 0.25 & 1 & 0 \\
\hline Patient 30 & 1.200 & 0.31 & 0.29 & 0.21 & 0.24 & 1 & 0 \\
\hline Patient 32 & 0.978 & 0.31 & 0.3 & 0.36 & 0.25 & 3 & 1 \\
\hline Patient 35 & 0.766 & 0.23 & 0.28 & 0.33 & 0.29 & 1 & 0 \\
\hline Patient 37 & 1.048 & 0.34 & 0.33 & 0.27 & 0.31 & 3 & 0 \\
\hline
\end{tabular}

DOI: 10.1136/annrheumdis-2017-eular.1462

\section{AB1025 THE SUPERB MICROVASCULAR IMAGING IS MORE SENSITIVE THAN CONVENTIONAL POWER DOPPLER IMAGING IN DETECTION OF ACTIVE SYNOVITIS IN RHEUMATOID ARTHRITIS PATIENTS}

G.Y. Lee $^{1}$, S. Kim ${ }^{1}$, S.T. Choi ${ }^{2}$, J.-S. Song ${ }^{2} .{ }^{1}$ Radiology; ${ }^{2}$ Internal Medicine, CHUng-Ang University College of Medicine, Seoul, Korea, Republic Of

Background: Precise evaluation of synovial inflammation and bony deformity is very important for the management of rheumatoid arthritis (RA). One of the most popular used methods to detect synovial inflammation and bony erosion is ultrasonography. Previous literatures revealed that US using power Doppler imaging (PDI) could detect more sensitive synovial inflammation than conventional radiography. However, there are still some limitations in ultrasonography. The superb microvascular imaging (SMI) is a new software technology introduced by Toshiba, which can detect a vascularity more sensitively without artifacts.

Objectives: In this prospective study, we evaluated the clinical usefulness of the SMI compared to PDI for the detection of active synovitis in patients with RA.

Methods: This prospective observational study includes 56 patients with RA (42 females; mean age,), from June 2015 to October 2016. The mean age of RA patients was $53.2 \pm 17.6$ years, and 42 patients were female $(75.0 \%)$. All the included patients underwent ultrasound about both wrists and hands (total 22 joints; wrist joints, metacarpophalangeal joints, and proximal interphalangeal joints). All the ultrasound examinations were performed at the volar side of the wrists and hands using both conventional PDI and SMI which use Aplio TM 500 Ultrasound (Toshiba Medical Systems Corporation). Their results were scored for each joint from grade 0 to grade 3 according to the vascularity (grade 0 , no vascularity; grade 1, single vessel; grade 2 , vascular flow less than $50 \%$ in field of view; grade 3 , equal to $50 \%$ or more). The sum of grades for 22 joints was compared between PDI (PDI-sum) and SMI (SMI-sum). The correlation between the sum of grades values and inflammatory laboratory parameters including the erythrocyte sedimentation rate (ESR), C-reactive protein (CRP), and disease activity score 28 (DAS28) were also evaluated.

Results: The mean values of ESR, CRP and DAS28 were $27.13 \pm 18.06 \mathrm{~mm} / \mathrm{hr}$, $6.78 \pm 9.14 \mathrm{mg} / \mathrm{L}$, and $2.71 \pm 1.11$ respectively. The positive rates of rheumatoid factor and anti-cyclic citrullinated antibody were $73.2 \%$ and $75.0 \%$, respectively. The sum of grades for 22 joints was significantly higher in SMI-sum compared to PDI-sum $(10.27 \pm 6.20$ vs. $5.80 \pm 3.79, p<0.001)$. The SMI-sum was highly correlated with the PDI-sum score $(\gamma=0.800, p<0.001)$. The SMI-sum showed positive correlation with DAS28, tender joint count, swollen joint count, visual analogue pain scale, and CRP level $(\gamma=0.486, p<0.001 ; \gamma=0.385, p=0.003$; $\gamma=0.467, \quad p<0.001 ; \gamma=0.547, p<0.001 ; \gamma=0.351, p=0.008$, respectively). The number of clinical remission (DAS28 score below 2.6) was $28(50.0 \%)$. The SMI-sum was significantly higher than PDI-sum in patients with clinical remission $(7.96 \pm 5.39$ vs. $4.64 \pm 3.03, p<0.001)$. All of the patients with clinical remission showed active synovitis at more than one joint in SMI.

Conclusions: SMI showed a more sensitive vascularity in RA patients than PDI. We could detect active synovitis through SMI in the RA patients with clinical remission. SMI could be a useful technology for the evaluation of synovitis in RA patients, especially for the detection of clinically subtle, but active synovitis in RA patients with remission.

Disclosure of Interest: None declared

DOI: 10.1136/annrheumdis-2017-eular.4571 